\title{
POLAR $\sigma$-IDEALS OF COMPACT SETS
}

\author{
GABRIEL DEBS
}

\begin{abstract}
Let $E$ be a metric compact space. We consider the space $\mathscr{H}(E)$ of all compact subsets of $E$ endowed with the topology of the Hausdorff metric and the space $\mathscr{M}(E)$ of all positive measures on $E$ endowed with its natural $w^{*}$-topology. We study $\sigma$-ideals of $\mathscr{K}(E)$ of the form $I=I_{P}=\{K \in$ $\mathscr{K}(E): \mu(K)=0, \forall \mu \in P\}$ where $P$ is a given family of positive measures on $E$.

If $M$ is the maximal family such that $I=I_{M}$, then $M$ is a band. We prove that several descriptive properties of $I$ : being Borel, and having a Borel basis, having a Borel polarity-basis, can be expressed by properties of the band $M$ or of the orthogonal band $M^{\prime}$.
\end{abstract}

Although this work will not use much Harmonic Analysis, its motivation goes back to the study of the interesting $\sigma$-ideals $U$ and $U_{0}$ on the torus $\mathbf{T}$. Recall that a compact subset $K$ of $\mathrm{T}$ is a $U$-set if and only if $K$ does not support any distribution $S$ such that $\lim _{n \rightarrow \infty}|\widehat{S}(n)|=0$ (where $\widehat{S}$ is the Fourier transform of $S$ ). The notion of compact $U_{0}$-set is obtained just by replacing in the last definition "distribution" by "measure" or equivalently by "positive measure". It has been well known since the fundamental work of Pyateskii-Sapiro that $U$ is a proper subset of $U_{0}$. However, recent results, of A. Kechris and A. Louveau (see [7]), and J. Saint Raymond and the author (see [1]) have shown some descriptive structural properties distinguishing these two $\sigma$-ideals. For example, $U_{0}$ has a Borel basis whereas $U$ does not. Another property-call it (*)-satisfied by $U_{0}$ and not by $U$ is the following: Given any Borel subset $A$ of $\mathrm{T}$, either $A$ is contained in the union of a countable family of compact $U_{0}$-sets or $A$ contains a compact set not in $U_{0}$. (For undefined notions and more details see $[1,4,7])$.

From now on, $E$ denotes a metric compact space and $\mathscr{K}(E)$ the space of all compact subsets of $E$ endowed with the Hausdorff topology. The properties of $U_{0}$ mentioned above are shared by the $\sigma$-ideal $\mathscr{K}_{\omega}(E)$ of all countable compact subsets of $E$. In fact, the family of all singletons forms a closed basis for $\mathscr{H}_{\omega}(E)$ and the property $(*)$ is just a reformulation of the classical "perfect set theorem" for Borel sets. The starting point of our work is the simple observation that, unlike $U$, both $\sigma$-ideals $U_{0}$ and $\mathscr{K}_{\omega}(E)$ are of the form $I=I_{P}=\{K \in \mathscr{K}(E): \mu(K)=0, \forall \mu \in P\}$ where $P$ is some fixed family of measures. This follows from the definition in the case of $U_{0}$, and

Received by the editors May, 19, 1993 and, in revised form, April 1, 1994.

1991 Mathematics Subject Classification. Primary 28A99, 46A55, 04A15; Secondary 42A63. 
for $I=\mathscr{K}_{\omega}(E)$ one can take for $P$ the set of all continuous measures. Our purpose in what follows is a systematic study of such $\sigma$-ideals (that we shall call polar $\sigma$-ideals). However, we shall quickly restrict our study to the case where $P$ is a Borel or more generally an analytic set in the space $\mathscr{M}(E)$ of all positive measures equipped with the vague topology.

In $\S 1$ we list briefly the main properties of general $\sigma$-ideals of compact sets. In $\S 2$ we discuss equivalent definitions and general properties of bands of measures. The notion of band is intimately linked to the notion of polar $\sigma$-ideal. In fact, if $I=I_{P}$ is a polar $\sigma$-ideal as above, then it is easy to see that there is a maximal family $M$ of measures such that $I=I_{M}$ and that family $M$ is a band. Actually it will be clear from our discussion that the notion of band is an analog of the notion of $\sigma$-ideal when we replace the ordered space $\mathscr{K}(E)$ by the ordered space $\mathscr{M}(E)$. However, as appears in $\S 3$, from a descriptive point of view, bands behave quite differently from $\sigma$-ideals; namely, we show that the dichotomy phenomenon (Kechris-Louveau-Woodin) does not occur for bands. Going back to the maximal band $M$ defining a given polar $\sigma$-ideal one can see that $M$ is also a strongly convex set. In the rest of the paper we focus on properties of bands which are Borel and strongly convex; we call such bands: standard bands.

In $\S 5$ we introduce the notion of polarity between families of measures and families of compact sets. In particular, we study "polarity basis" (or simply " $p$-basis") for an arbitrary $\sigma$-ideal $I$ : This is a subset $B$ of $I$ such that any measure which is null on $B$ is automatically null on $I$. Any basis is obviously a $p$-basis, and most of the structural properties of basis are also true for $p$ basis. We give several examples showing that these notions are distinct. We also discuss in this section a theorem of $\mathrm{G}$. Mokobodzki which will play a central role in the rest of our work.

The first main result is proved in $\S 6$ and asserts that if $I$ is a polar $\sigma$-ideal defined by an analytic family $P$ of measures, $M$ the maximal band defining $I$ and $M^{\prime}$ the orthogonal band of $M$, then the following conditions are equivalent:

(i) $I$ has a Borel $p$-basis.

(ii) $M$ is Borel.

(iii) $M^{\prime}$ is Borel.

The proof makes extensive use of the Effective Descriptive Set Theory. A brief and heuristic presentation of this theory can be found in the preleminaries that we give in $\S 0$. In $\S \S 7$ and 8 we discuss the particular cases where $I$ is Borel or has a Borel basis.

The second main result is proved in $\S 9$ and gives a full description of all standard bands: Any Borel strong band $M$ has a representation of the form $M=\left\{\mu: \lim _{\mathscr{F}} \mu\left(f_{n}\right)=0\right\}$ where $\left(f_{n}\right)$ is a sequence of continuous functions on $E$ with $0 \leq f_{n} \leq 1$ and $\mathscr{F}$ is a copy on $\omega$ of an iterated Fréchet filter. Moreover, we can choose this representation so that $M^{\prime}=\left\{\mu: \varlimsup_{\mathscr{I}} \mu\left(f_{n}\right)=\right.$ $\mu(1)\}$.

We finish the paper by listing in $\S 10$ several natural problems which arise form the study of polarity. One of them (Question 7) was solved by the referee, and we are grateful to him for communicating to us the solution that we reproduce in this section. 


\section{Preliminaries}

0.1. General notation. Throughout this paper $E$ denotes a fixed compact metric space.

$\mathscr{K}(E)$ or simply $\mathscr{K}$ will denote the set of all closed subsets of $E$ and will be equipped with the Hausdorff topology. We recall that this topology is compact and metrizable (for example, by the Hausdorff distance) and is generated by the sets of the two following forms: $\{K \in \mathscr{K}: K \subset V\}$ and $\{K \in \mathscr{K}: K \cap V \neq \varnothing\}$ for $V$ an arbitrary open subset of $E$.

By a measure on $E$ we shall always mean a positive Radon measure. The set of all measures on $E$ will be denoted by $\mathscr{M}(E)$ or simply $\mathscr{M}$. On the Banach space $\mathscr{M}-\mathscr{M}$ of all signed measures on $E$ we shall consider both the norm topology and the vague topology (i.e., the $w^{*}$-topology). However, unless there is some explicit indication otherwise, all topological and descriptive notions considered on $\mathscr{M}(E)$ are relative to the vague topology; we recall that for this topology $\mathscr{M}$ is separable, metrizable, and locally compact space.

0.2. Classical descriptive classes. Let $X$ be some fixed Polish space (in fact, we shall apply what follows to $X=E, \mathscr{K}(E)$, or $\mathscr{M}(E)$ ).

We shall consider on $X$ the Borel additive and multiplicative classes $\Sigma_{\xi}^{0}(X)$ and $\Pi_{\xi}^{0}(X)$. We recall that these classes are defined inductively for the countable ordinal $\xi \geq 1$ in the following way:

$$
\left\{\begin{array}{l}
\Sigma_{1}^{0}(X) \text { is the class of all open subsets of } X, \\
\Pi_{1}^{0}(X) \text { is the class of all open subsets of } X ;
\end{array}\right.
$$

and for $\xi>1$ :

$$
\left\{\begin{array}{l}
\Sigma_{\xi}^{0}(X)=\left\{\bigcup_{n \in \omega} A_{n} ; \text { with } A_{n} \in \bigcup_{\eta<\xi} \Pi_{\eta}^{0}(X) \text { for all } n\right\}, \\
\Pi_{\xi}^{0}(X)=\left\{\bigcup_{n \in \omega} A_{n} ; \text { with } A_{n} \in \bigcup_{\eta<\xi} \Sigma_{\eta}^{0}(X) \text { for all } n\right\} .
\end{array}\right.
$$

In particular, $\Pi_{2}^{0}(X)=\mathbf{G}_{\delta}(X)$, and if the space $X$ is $\mathbf{K}_{\sigma}$, then $\Sigma_{2}^{0}(X)=$ $\mathbf{K}_{\sigma}(X)$.

We shall also consider the classes:

$$
\left\{\begin{array}{l}
\Sigma_{1}^{1}(X): \text { the class of all analytic subsets of } X, \\
\Pi_{1}^{1}(X): \text { the class of all coanalytic subsets of } X, \\
\Delta_{1}^{1}(X): \text { the class of all Borel subsets of } X .
\end{array}\right.
$$

Let $\Gamma$ denote any of the previous classes aside the three classes $\Pi_{1}^{0}, \Sigma_{1}^{0}, \Sigma_{2}^{0}$, and suppose that a set $A$ is homeomorphically embedded in two Polish spaces $X$ and $X^{\prime}$. Then $A \in \Gamma(X)$ if and only if $A \in \Gamma\left(X^{\prime}\right)$; we shall then say that $A$ as in $\Gamma$ without specifying the reference space $X$.

For $\Sigma_{2}^{0}$ we have a similar absoluteness property if we restrict the reference space $X$ to be a $\mathbf{K}_{\sigma}$ space, and this will be the case for most of the spaces that we shall consider in this work. To emphasize this fact, when the reference space is $\mathbf{K}_{\sigma}$ we shall use the notation $\mathbf{K}_{\sigma}$ rather than $\Sigma_{2}^{0}$.

0.3. Effective descriptive classes. Although all the results established in this work have classical statements, the proofs of some of them will use the Effective Descriptive Set Theory. For the reader who is not familiar with this theory we 
sketch here a quick presentation of its basic concepts, and we refer to [10] or [15] for a complete exposition.

First we insist that this theory does not involve any supplementary axioms to Set Theory. In particular, there is no restriction in the application of these results.

Effective Descriptive Set Theory concerns any Polish space equipped with a countable basis of the topology given by some fixed enumeration and satisfying a few technical restrictions: for example, $\mathbb{R}$ with any reasonable enumeration of all open intervals with rational end points. The first basic fact is that inside each classical class $\Gamma$ :

$$
\Sigma_{\xi}^{0}, \Pi_{\xi}^{0}, \Delta_{1}^{1}, \Sigma_{1}^{1}, \Pi_{1}^{1}
$$

one can define some subclass $\Gamma$ which can be considered heuristically as the class of all sets in $\Gamma$ for which one can show in an "effective" way that they belong to $\Gamma$. The mathematical formulation of these ideas is based on the notion of recursivity. These classes are called the effective classes and are denoted by

$$
\Sigma_{\xi}^{0}, \Pi_{\xi}^{0}, \Delta_{1}^{1}, \Sigma_{1}^{1}, \Pi_{1}^{1} .
$$

Each of these classes is countable but has similar stability properties as the corresponding classical class. For example, although $\Delta_{1}^{1}$ is not a $\sigma$-field, one can show that if $\left(A_{n}\right)$ is a " $\Delta_{1}^{1}$-sequence" of $\Delta_{1}^{1}$ sets, then $\bigcap_{n} A_{n}$ and $\bigcup_{n} A_{n}$ are also $\Delta_{1}^{1}$ sets.

Finally given a parameter $\alpha$ in some "reasonable" fixed space (for example, in $2^{\omega}$ or $\omega^{\omega}$ or $X$ itself) one can also define similar classes denoted by

$$
\Sigma_{\xi}^{0}(\alpha), \Pi_{\xi}^{0}(\alpha), \Delta_{1}^{1}(\alpha), \Sigma_{1}^{1}(\alpha), \Pi_{1}^{1}(\alpha) .
$$

The class $\Gamma(\alpha)$ can be considered as the class of all sets in $\Gamma$ for which, using all information coded by $\alpha$, one can show in an "effective" way that they belong to $\Gamma$ : For example, if $\alpha \in X$, then $\{\alpha\} \in \Gamma(\alpha)$. Then the second basic fact of the theory which enables one to derive from the properties of the effective classes similar properties for the classical classes is the formula

$$
\Gamma=\bigcup_{\alpha} \Gamma(\alpha) .
$$

\section{1. $\sigma$-IDEALS OF COMPACT SETS}

A subset $J$ of $\mathscr{K}$ is said to be hereditary, if for any $K$ and $L \in \mathscr{K}$ we have $((K \in J$ and $L \subset K) \Rightarrow(L \in J))$. A subset of $\mathscr{K}$ is an ideal if it is hereditary and stable by finite unions. A subset $I$ of $\mathscr{K}$ is a $\sigma$-ideal if it is hereditary and stable by compact countable unions, in other words, if for any $\left(K_{n}\right)_{n \in \omega} \subset I$ and any $K \in \mathscr{K}$, if $K \subset \bigcup_{n \in \omega} K_{n}$, then $K \in I$.

The descriptive properties of $\sigma$-ideals of compacts sets were extensively studied by A. Louveau, A. Kechris, and H. Woodin in [8]. The following results are mainly from [8] and shall be used freely in this work (1.2 is due to DoughertyKechris, Louveau; see [6]).

1.1. If $I$ is $\Sigma_{1}^{1}$ (i.e., analytic) $\sigma$-ideal, then $I$ is necessarily $\Pi_{2}^{0}$ (i.e., $\mathbf{G}_{\delta}$ ) . Actually most of the interesting examples of $\sigma$-ideals happen to appear as $\Pi_{1}^{1}$ (i.e., coanalytic) subsets in $\mathscr{K}$. Hence for a $\Pi_{1}^{1} \sigma$-ideal $I$ we have the following dichotomy: either $I$ is $\mathbf{G}_{\delta}$, or $I$ is non-Borel. 
1.2. If $I$ is a $\mathbf{G}_{\delta}$ ideal, then $I$ is a $\sigma$-ideal.

1.3. A basis for a $\sigma$-ideal $I$ is a subset $B \subset I$ such that any $K \in I$ can be covered by a countable family from $B$, that is, $K \subset \bigcup_{n \in \omega} K_{n}$ with $K_{n} \in B$ for all $n$. This is equivalent to saying that $I$ is the smallest $\sigma$-ideal containing $B$.

There are natural and interesting examples of non-Borel $\sigma$-ideals with a Borel basis. Here are some general properties of a basis:

(a) If $I$ has a $\boldsymbol{\Sigma}_{1}^{1}$ basis, then $I$ has a Borel (and even a $\mathbf{G}_{\delta}$ ) basis.

(b) If $I$ has a hereditary Borel basis, then $I$ is $\Pi_{1}^{1}$.

(c) If $I$ is $\Pi_{1}^{1}$ and has a Borel basis, then $I$ has a Borel basis which is an ideal.

1.4. A trivial but fundamental example of a $\sigma$-ideal is given by $\mathscr{K}(A)$, the $\sigma$-ideal of all compact subsets of a fixed set $A \subset E$. For such a $\sigma$-ideal we have:

(a) $\mathscr{K}(A)$ is $\boldsymbol{\Pi}_{1}^{1}$ if and only if $A$ is $\boldsymbol{\Pi}_{1}^{1}$.

(b) $\mathscr{K}(A)$ is $\mathbf{G}_{\delta}$ if and only if $A$ is $\mathbf{G}_{\delta}$.

(c) $\mathscr{K}(A)$ has a Borel basis if and only if $A$ is of the form $A=A_{1} \cap A_{2}$ with $A_{1}$ a $\mathbf{G}_{\delta}$ subset of $E$ and $A_{2}$ a $\mathbf{K}_{\sigma}$ subset of $E$.

\section{BANDS OF MEASURES}

We consider on $\mathscr{M}$ the natural ordering $\leq$. The inf and sup operations will be denoted by $\wedge$ and $\vee$. A subset $P$ of $\mathscr{M}$ is hereditary if for any $\mu$ and $\mu^{\prime} \in \mathscr{M}$ we have $\left(\left(\mu \in P\right.\right.$ and $\left.\left.\mu^{\prime} \leq \mu\right) \Rightarrow\left(\mu^{\prime} \in P\right)\right)$. A subset $P$ of $\mathscr{M}$ is said to be bounded for the order in $\mathscr{M}$ if there exists $\mu \in \mathscr{M}$ such that $\nu \leq \mu$ for any $\nu \in P$. We recall that any subset of $\mathscr{M}$ that is bounded for the order admits a supremum. A subset $P$ of $\mathscr{M}$ is complete for the order if the supremum of any bound for the order subset of $P$ belongs to $P$.

Proposition 2.1. For a subset $M$ of $\mathscr{M}$ the following conditions are equivalent:

(i) $M$ is a convex cone which is hereditary and complete for the order.

(ii) $M$ is a convex cone which is hereditary for the order and norm closed.

(iii) For any $\left(\mu_{n}\right)_{n \in \omega}$ in $M$ such that $\sum_{n \in \omega} \mu_{n}$ is defined in $\mathscr{M}$, and for any $\mu \in \mathscr{M}$, if $\mu \leq \sum_{n \in \omega} \mu_{n}$, then $\mu \in M$.

Proof. (i) $\Rightarrow$ (ii) If $\mu=\lim _{n \rightarrow \infty} \mu_{n}$ with $\left(\mu_{n}\right)_{n \in \omega} \subset M$, then by (i) the measures $\mu_{n}^{\prime}=\sup _{k \geq n}\left(\mu_{k} \wedge \mu\right)$ are well defined and $\mu_{n}^{\prime} \in M$. Then $\mu_{n} \wedge \mu \leq$ $\mu_{n}^{\prime} \leq \mu$ and it follows from the norm continuity of the inf operation $\wedge$ that $\mu=\lim _{n \rightarrow \infty} \mu_{n}^{\prime}$; and since the sequence $\left(\mu_{n}^{\prime}\right)_{n \in \omega}$ is increasing, we have $\mu=$ $\sup _{n \in \omega} \mu_{n}^{\prime}$, so $\mu \in M$.

(ii) $\Rightarrow$ (iii) obvious.

(iii) $\Rightarrow$ (i) It is easy to deduce from (iii) that $M$ is a hereditary convex cone. Let $P \subset M$, and suppose $P$ bounded for the order; then there exists $\left(\lambda_{n}\right)_{n \in \omega} \subset P$ so that $\lambda=\sup (P)=\sup _{n \in \omega} \lambda_{n}$. Since (iii) implies that $M$ is stable by taking finite $\wedge$ and $\vee$, we can find an increasing sequence $\left(\nu_{n}\right)_{n \in \omega}$ in $M$ such that $\lambda=\sup _{n \in \omega} \nu_{n}$ such that $\lambda=\sum_{n \in \omega} \mu_{n}$ with $\mu_{0}=\nu_{0}$ and $\mu_{n+1}=\nu_{n+1}-\nu_{n}$ for any $n \geq 0$.

2.2. Bands. A subset $M$ of $\mathscr{M}$ is a band if it satisfies one of the equivalent conditions of 2.1. Notice the formal similarity between the notion of $\sigma$-ideal in $\mathscr{K}$ and the notion of band as expressed in (iii). 
One can also consider on $\mathscr{M}$ the weaker order $<<$ of absolute continuity. Then it is clear that if we replace in (ii) hereditarity for $\leq$ by hereditarity for $<<$, we obtain also an equivalent condition.

Many authors define a band as a vector subspace $V$ of $\mathscr{M}-\mathscr{M}$ satisfying conditions similar to those of Proposition 2.1 from which it follows that $V^{+}=$ $V \cap \mathscr{M}$ satisfies our definition and that $V$ is of the form $V=V^{+}-V^{+}$. Such vector spaces $V$ are also called $L$-spaces.

2.3. Operations on bands. If $\left(M_{i}\right)_{i \in I}$ is any family of bands, then $\bigcap_{i \in I} M_{i}$ is a band. The band generated by $\bigcup_{i \in I} M_{i}$ (i.e., the smallest band containing $\left.\bigcup_{i \in I} M_{i}\right)$ is denoted by $\sum_{i \in I} M_{i}$.

2.4. Generation of bands. Let $P$ be any subset of $\mathscr{M}$. Then:

(a) The hereditary convex cone generated by $P$ is the set

$$
H=\left\{\mu \in \mathscr{M}: \mu \leq \sum_{i=1}^{n} \alpha_{i} \mu_{i}, \alpha_{i} \geq 0, \mu_{i} \in P, n \in \omega\right\} .
$$

(b) The band $M$ generated by $P$ is the norm closure of $H$. More precisely if for any $\varepsilon>0$ we let $\mathscr{M}_{\varepsilon}=\{\mu \in \mathscr{M}:\|\mu\| \leq \varepsilon\}$, then $M=\bigcap_{\varepsilon>0}\left(H+\mathscr{M}_{\varepsilon}\right)$.

2.5. Examples. In the sequel we shall refer frequently to the following examples:

(1) For any subset $A \subset E$ :

$\mathscr{M}^{*}(A)$ is the band of all measures $\mu$ outerly supported by $A$ (i.e., $\mu^{*}(A)=$ $\mu(E))$ and

$\mathscr{M}_{*}(A)$ is the band of all measures $\mu$ innerly supported by $A$ (i.e., $\mu_{*}(A)=$ $\mu(E))$.

When $A$ is universally measurable we shall write $\mathscr{M}(A)$ for $\mathscr{M}_{*}(A)=$ $\mathscr{M}^{*}(A)$.

(2) $\mathscr{M}_{c}(E)$ is the band of all continuous measures on $E$ and

$\mathscr{M}_{d}(E)$ is the band of all discrete measures on $E$.

(3) Let $\left(f_{n}\right)_{n \in \omega}$ be a uniformly bounded sequence of nonnegative and continuous functions on $E$ and let $\mathscr{F}$ be some filter on $\omega$. Then $M=\{\mu \in$ $\left.\mathscr{M}: \lim _{\mathscr{G}} \mu\left(f_{n}\right)=0\right\}$ is a band.

(4) Let $E=\mathbf{T}$ be the torus, and let $\hat{\mu}$ denote the Fourier transform of any $\mu \in \mathscr{M}$. Then the set $M_{0}(\mathbf{T})=\left\{\mu \in \mathscr{M}: \lim _{n \rightarrow \infty} \hat{\mu}(n)=0\right\}$ of all Rajchman measures is a band. This is not a particular case of example (3) but follows from the algebraic properties of the functions $f_{n}$; see [4, p. 104, Lemma 4.4.1] or [7, p. 77, Lemma II.5.4].

2.6. Orthogonal bands. We denote as usual by $\perp$ the orthogonality relation between measures. We recall some elementary facts. Given any subset $P$ of $\mathscr{M}$ the set $P^{\prime}=\{\mu \in \mathscr{M}: \mu \perp \nu, \forall \nu \in P\}$ is a band called the orthogonal band of $P$. Now if we repeat this operation on $P^{\prime}$ we obtain a band $\left(P^{\prime}\right)^{\prime}$ which is exactly the band generated by $P$. In particular, if $M$ is a band, then $\left(M^{\prime}\right)^{\prime}=M$ and the bands $M$ and $M^{\prime}$ are mutually orthogonal. Moreover, any $\lambda \in \mathscr{M}$ has a unique decomposition $\lambda=\mu+\mu^{\prime}$ with $\mu \in M$ and $\mu^{\prime} \in M^{\prime}$.

In the preceding examples we have:

(1) $\mathscr{M}^{*}(A)$ and $\mathscr{M}_{*}\left(A^{c}\right)$ are mutually orthogonal bands.

(2) $\mathscr{M}_{c}(E)$ and $\mathscr{M}_{d}(E)$ are mutually orthogonal bands.

(3) For a description of $M^{\prime}$ when $M=M_{0}$ (T) see [14]. 


\section{DESCRIPTIVE PROPERTIES OF BANDS}

The main purpose of this section is to show that unlike for $\sigma$-ideals, there is no dichotomy phenomena for the possible descriptive classes of bands.

Proposition 3.1. Let $\Gamma$ be any of the classes $\Pi_{\xi}^{0}, \Sigma_{1}^{1}, \Pi_{1}^{1}$. Then $\mathscr{M}(A)$ is in $\Gamma(\mathscr{M})$ if and only if $A$ is in $\Gamma(E)$.

Proof. Let $\Delta=\left\{\delta_{x} ; x \in E\right\}$ be the set of all Dirac measures on $E$. Since $A$ is homeomorphic to $\mathscr{M}(A) \cap \Delta$ and since al the considered classes are stable by taking the intersection with a closed set, the condition is clearly necessary. For sufficiency we distinguish three cases.

(1) Suppose $A$ is $\Pi_{\xi}^{0}$ and observe that

$$
\mu \in \mathscr{M}(A) \Leftrightarrow\left(\mu \neq 0 \text { and } \frac{1}{\mu(E)} \mu(A)=1\right) \text { or }(\mu=0) .
$$

Since the mapping $\mu \mapsto \frac{1}{\mu(E)} \mu$ is continuous on $\mathscr{M} \backslash\{0\}$, the result is a consequence of the following fact which can be checked easily by induction on $\xi$.

Fact. If $A$ is $\Pi_{\xi}^{0}$, then for any $\alpha \geq 0$ the set $\{\mu \in \mathscr{M}: \mu(A) \geq \alpha\}$ is $\Pi_{\xi}^{0}$.

(2) Suppose $A$ is $\Sigma_{1}^{1}$. Fix some Polish space $P$ and a continuous surjection $\varphi: P \rightarrow A$. Then the canonical extension $\bar{\varphi}: \mathscr{M}(P) \rightarrow \mathscr{M}(A)$ of $\varphi$ (which assigns to any $\lambda \in \mathscr{M}(P)$ the measure $\varphi(\lambda)$ on $A)$ is continuous, and it is well known that $\bar{\varphi}$ is also onto (this follows, for example, from the Choquet capacitability theorem). Since $\mathscr{M}(P)$ is Polish by case $(1), \mathscr{M}(A)$ is $\Sigma_{1}^{1}$.

(3) Suppose $A$ is $\Pi_{1}^{1}$. Then observing that

$$
\mu \notin \mathscr{M}(A) \Leftrightarrow \exists \nu \in \mathscr{M}\left(A^{c}\right): \nu \neq 0, \nu \leq \mu
$$

it follows from case (2) that $\mathscr{M}(A)$ is $\Pi_{1}^{1}$.

As we shall see the previous result is not true for $\Gamma=\Sigma_{\xi}^{0}$. In fact, the next result shows that the natural scale for comparing complexity of Borel bands is given by the multiplicative classes $\Pi_{\xi}^{0}$ rather than the additive classes $\Sigma_{\xi}^{0}$.

Proposition 3.2. If a band $M$ is $\boldsymbol{\Sigma}_{\xi}^{0}$, then necessarily $M$ is $\Pi_{\eta}^{0}$ for some $\eta<\xi$.

Proof. We shall prove that $N=M \backslash\{0\}$ is $\Pi_{\eta}^{0}$ for some $\eta<\xi$, from which will follow the same conclusion for $M$. Let $X$ denote the Polish space $\mathscr{M} \backslash\{0\}$, and consider the continuous mapping $\Phi: X^{\omega} \rightarrow X$ defined by

$$
\Phi\left(\left\langle\mu_{n}\right\rangle_{n \in \omega}\right)=\sum_{n=0}^{\infty} \frac{1}{2^{n}\left\|\mu_{n}\right\|} \mu_{n}
$$

Fix $\left\langle\mu_{n}\right\rangle_{n \in \omega}$ in $X^{\omega}$, and let $\mu=\Phi\left(\left\langle\mu_{n}\right\rangle_{n \in \omega}\right):$ If $\left\langle\mu_{n}\right\rangle_{n \in \omega} \in N^{\omega}$, then, since $M$ is convex and norm closed, we have $\mu \in N$. Conversely if $\mu \in N$, then, since $M$ is hereditary and $\mu_{n} \leq 2^{n}\left\|\mu_{n}\right\| \mu$, we have $\left\langle\mu_{n}\right\rangle_{n \in \omega} \in N^{\omega}$. Hence $N^{\omega}=\Phi^{-1}(N)$.

Let $\mathscr{W}$ denote the Wadge class of $N$, that is, the class of all subsets of $2^{\omega}$ of the form $\varphi^{-1}(N)$ for some continuous mapping $\varphi: 2^{\omega} \rightarrow X$. It follows 
from the properties of $\Phi$ that $\mathscr{W}$ is stable by countable intersections: If $\left(A_{n}\right)$ is a sequence in $\mathscr{W}$ with $A_{n}=\varphi_{n}^{-1}(N)$, then $\bigcap_{n} A_{n}=\varphi^{-1}(N)$ with $\varphi$ defined by $\varphi(\alpha)=\Phi\left(\left\langle\varphi_{n}(\alpha)\right\rangle_{n \in \omega}\right)$.

Now we shall use the following general basic fact about Wadge's classes (see [12] for more details):

Fact. Let $N$ be a Borel subset of some Polish space $X$, and let $\mathscr{W}$ be the Wadge class of $N$. If $N \notin \Pi_{\eta}^{0}(X)$, then $\mathscr{W} \supset \Sigma_{\eta}^{0}\left(2^{\omega}\right)$.

So suppose by contradiction that $N \notin \bigcup_{\eta<\xi} \Pi_{\eta}^{0}(X)$. Then $\mathscr{W} \supset \bigcup_{\eta<\xi} \Sigma_{\eta}^{0}\left(2^{\omega}\right)$ and since in this case $\mathscr{W}$ is stable by countable intersections, $\mathscr{W} \supset \Pi_{\xi}^{0}\left(2^{\omega}\right)$. But on the other hand, since $M$ is supposed by hypothesis to be $\Sigma_{\xi}^{0}$, we also have $\mathscr{W} \subset \Sigma_{\xi}^{9}\left(2^{\omega}\right)$, from which we can derive that $\Pi_{\xi}^{0}\left(2^{\omega}\right) \subset \Sigma_{\xi}^{0}\left(2^{\omega}\right)$, which is false.

Let $A$ be a $\Sigma_{\xi}^{0}$ set in $E$; then by Proposition $3.1 \mathscr{M}(A)$ is $\Pi_{\xi+1}^{0}$. Conversely if $\mathscr{M}(A)$ is $\Sigma_{\xi+1}^{0}$, then it follows from Propositions 3.2 that $\mathscr{M}(A)$ is $\Pi_{\xi}^{0}$ and again from Proposition 3.1 that $A$ is also $\Pi_{\xi}^{0}$. Thus if $A$ is $\Sigma_{\xi}^{0} \backslash \Pi_{\xi}^{0}$, then $\mathscr{M}(A)$ is $\Pi_{\xi+1}^{0} \backslash \Sigma_{\xi+1}^{0}$. The same arguments show that if $A$ is $\Pi_{\xi}^{0} \backslash \Sigma_{\xi}^{0}$, then $\mathscr{M}(A)$ is $\boldsymbol{\Pi}_{\xi}^{0} \backslash \Sigma_{\xi}^{0}$.

3.3. Analytic bands. In this work we shall consider mainly analytic bands, for which we list here some basic properties. Let $M$ be a band and $M^{\prime}$ its orthogonal band:

(a) If $M$ is generated by an analytic subset, then $M$ is analytic.

(b) If $M$ is separated from $M^{\prime}$ by an analytic subset $S$ (i.e., $M \subset S$ and $\left.S \cap M^{\prime}=\{0\}\right)$-in particular, if $M$ is analytic-then $M^{\prime}$ is coanalytic.

(c) The following conditions are equivalent:

(i) $M$ and $M^{\prime}$ are Borel.

(ii) $M$ and $M^{\prime}$ are analytic.

(iii) $M$ and $M^{\prime}$ are separated by a Borel set.

(a) follows immediately from 2.3 . For (b) notice that

$$
\lambda \notin M^{\prime} \Leftrightarrow \exists \mu \in S, \mu \neq 0, \mu \leq \lambda .
$$

Finally (c) follows from (b) and the separation theorem for analytic sets.

\section{STRONG CONVEXITY OF BANDS}

4.1. Strong convexity. The notion of strongly convex subset of a fixed compact convex set is well known (see [3, p. 186], or [7, Chapter IX]). However, in our study the compactness restriction is artificial and we discuss here briefly this notion for subsets of $\mathscr{M}$. Although we shall see that the general case can be reduced to the compact case, such a reduction is not always convenient and might create unpleasant formal complications.

(a) If $\pi$ is a probability measure on an abstract measurable space $(T, \mathscr{T})$, we shall say that the mapping $\varphi: T \rightarrow \mathscr{M}$ is integrable whenever $\int\|\varphi(t)\| d \pi(t)<$ $+\infty$. Notice that since we are dealing only with nonnegative measures on a compact space, the last condition is equivalent to saying that $\int\langle\varphi(t), f\rangle d \pi(t)<$ $+\infty$ for any continuous real valued function $f$ on $E$. The integral $\int \varphi(t) d \pi(t)$ is then an element of $\mathscr{M}$. 
(b) If $\pi$ is a (Radon) probability on $\mathscr{M}$, the barycenter of $\pi$ is defined if the identity map of $\mathscr{M}$ is integrable; we denote $b(\pi)=\int \lambda d \pi(\lambda)$.

(c) For a subset $C$ of $\mathscr{M}$ the following conditions are equivalent:

(i) For any compact subset $K$ of $C$, the closed convex hull of $K$ is contained in $C$.

(ii) For any probability $\pi$ on $\mathscr{M}$ innerly supported by $C$, if the barycenter $b(\pi)$ is defined, then $b(\mu) \in C$.

(iii) For any probability $\pi$ on an abstract measurable space $(T, \mathscr{T})$ and any measurable mapping $\varphi: T \rightarrow C$, if $\varphi$ is integrable, then the integrable $\int \varphi(t) d \pi(t)$ is an element $C$.

(iii) $\Leftrightarrow$ (ii) $\Rightarrow$ (i) are obvious. Minor modifications of [3, p. 186, Theorem] or $[7$, p. 311 , Lemma 1] give (i) $\Rightarrow$ (ii) .

Notice that it follows from condition (i) that $C$ is strongly convex if and only if $C \cap \mathscr{M}_{r}$ is strongly convex for any $r>0$.

4.2. Strong bands. A subset $M$ of $\mathscr{M}$ will be said to be a strong band if $M$ is a band and a strongly convex set. Here are some examples:

(1) For any set $A \subset E$ the band $\mathscr{K}^{*}(A)$ is strong. To see this notice that for any $\lambda \in \mathscr{M}$ we have

$$
\lambda \in \mathscr{M}^{*}(A) \Leftrightarrow \lambda(K)=0, \forall K \text { compact } \subset A^{c}
$$

and apply condition (ii) of 4.1 (c).

(2) $\mathscr{M}_{c}(E)$ is a strong band, but $\mathscr{M}_{d}(E)$ is not a strong band.

(3) $M_{0}(\mathrm{~T})$ is a strong band. This is an immediate consequence of the Lebesgue dominated convergence theorem.

4.3. Orthogonal band of a strong band. It follows from example (1) of 4.2 that if $A$ is a universally measurable subset of $E$ then both bands $M=\mathscr{M}(A)$ and $M^{\prime}=\mathscr{M}\left(A^{c}\right)$ are strong bands. However this is almost the unique example of such a situation. In fact for any band $M$ if we let $A=\left\{x \in E: \delta_{x} \in M\right\}$ and $A^{\prime}=\left\{x \in E: \delta_{x} \in M^{\prime}\right\}$, then it is clear that necesarily $A^{\prime}=A^{c}$. So if we suppose that $M$ and $M^{\prime}$ are strong bands then we obtain that $\mathscr{M}_{*}(A) \subset M$ and $\mathscr{M}_{*}\left(A^{c}\right) \subset M^{\prime}$, so $\mathscr{M}_{*}(A) \subset M \subset \mathscr{M}^{*}(A)$. If we suppose moreover that $M$ is universally measurable then the set $A$ which is of the form $A=\Phi^{-1}(M)$ for some continuous mapping $\Phi: E \rightarrow \mathscr{M}$ is also universally measurable and hence $M=\mathscr{M}(A)$.

We now consider the problem of the generation of strong bands. For an arbitrary subset $P$ of $\mathscr{M}$ there is no simple inner description of the sgrong band generated by $P$, similar to 2.3 . But as we shall see, this is possible if $P$ is an analytic subset of $\mathscr{M}$. The following result, for which we give here an elementary proof, can be obtained as a by-product of the proof of a deep Theorem of Mokobodzki that we shall discuss later.

Theorem 4.4. Let $P$ be an analytic subset of $\mathscr{M}$.

(a) The strongly convex set $Q$ generated by $P$ is the set of all defined barycenters of probabilities supported by $P$. In particular, $Q$ is analytic.

(b) The strong band $M$ generated by $P$ is the band generated by $Q$. In particular, $M$ is analytic.

Proof. (a) Let $\mathscr{P}$ be the set of all probabilities $\pi$ on $\mathscr{M}$ such that $b(\pi)$ is defined and $\pi(P)=1$. Let $\mathscr{E}$ be any metric compactification of $\mathscr{M}$. Then 


$$
\begin{gathered}
\mathscr{P}=\mathscr{M}(P) \cap \mathscr{B} \text { with } \mathscr{B}=\mathscr{B}_{0} \cap \mathscr{B}_{1} \text { and } \\
\pi \in \mathscr{B}_{0} \Leftrightarrow b(\pi) \text { is defined } \Leftrightarrow \int_{\mathscr{M}} \mu(1) d \pi(\mu)<+\infty, \\
\pi \in \mathscr{B}_{1} \Leftrightarrow \pi(1)=1 .
\end{gathered}
$$

It is easy to see that $\mathscr{B}$ is a Borel subset of $\mathscr{M}(\mathscr{E})$ and hence by 3.1 that $\mathscr{P}$ is an analytic subset of $\mathscr{M}(\mathscr{E})$. So $Q^{\prime}=b(\mathscr{P})$ is analytic and $P \subset Q^{\prime} \subset Q$. To finish the proof and to show that $Q^{\prime}=Q$ it is sufficient to prove that $Q^{\prime}$ is strongly convex.

So let $\rho$ be a probability on $Q^{\prime}$ such that $b(\rho)$ is defined; we shall prove that $b(\rho) \in Q^{\prime}$. Since the mapping $b: \mathscr{P} \rightarrow Q^{\prime}$ is onto and Borel (not necessarily continuous if $P$ is not relatively compact!), by the Jankov-von Neumann cross section theorem we can find a $\rho$-measurable inverse $\lambda \mapsto \pi_{\lambda}$ from $Q^{\prime}$ into $\mathscr{P}$ such that $b\left(\pi_{\lambda}\right)=\lambda$ for any $\lambda \in Q^{\prime}$. Now if we define $\pi=\int \pi_{\lambda} d \rho(\lambda)$ then an immediate computation shows that $\pi \in \mathscr{P} ;$ moreover, $b(\rho)=\int \lambda d \rho(\lambda)=$ $\int b\left(\pi_{\lambda}\right) d \rho(\lambda)=b(\pi)$ so that $b(\rho) \in Q^{\prime}$.

(b) Let $N$ be the band generated by $Q$. Then $P \subset Q \subset N \subset M$, and it is sufficient to prove that $N$ is strongly convex. Let $\widetilde{Q}=\{\mu \in \mathscr{M}: \exists \nu \in Q, \mu \leq \nu\}$ then $\widetilde{Q}$ is a hereditary strongly convex set and $Q \subset \widetilde{Q} \subset N$. So $H=\bigcup_{n \in \omega} n \widetilde{Q}$ is a hereditary convex cone and by $(2.3)$ we have $N=\bigcap_{\varepsilon>0}\left(H+\mathscr{M}_{\varepsilon}\right)$.

Let $\pi$ be a probability supported by $N \cap \mathscr{M}_{1}$ and fix $0<\varepsilon \leq 1$. For any $n \in \omega$ let $H_{n}=n \widetilde{Q} \cap \mathscr{M}_{1}$. Since $\pi$ is supported by $\left[\bigcup_{n \in \omega} H_{n}+\mathscr{M}_{\varepsilon}\right] \subset \mathscr{M}_{2}$, we can write $\pi=\alpha_{n} \pi_{n}^{\prime}+\left(-1 \alpha_{n}\right) \pi_{n}^{\prime \prime}$ with $\pi_{n}^{\prime}$ and $\pi_{n}^{\prime \prime}$ probabilities supported by $\left(H_{n}+\mathscr{M}_{\varepsilon}\right)$ and $\left(H_{n}+\mathscr{M}_{\varepsilon}\right)^{c}$ such that $\alpha_{n}=\pi\left(H_{n}+\mathscr{M}_{\varepsilon}\right)$ satisfies $\lim _{n \rightarrow \infty} \alpha_{n}=1$. Since $b(\pi)=\alpha_{n} b\left(\pi_{n}^{\prime}\right)+\left(1-\alpha_{n}\right) b\left(\pi_{n}^{\prime \prime}\right)$ we an fix $n$ such that $\left\|b(\pi)-\alpha_{n} b\left(\pi_{n}^{\prime}\right)\right\| \leq$ $\varepsilon$. But it is easy to see that $H_{n}+\mathscr{M}_{\varepsilon}$ (subset of the compact convex set $\mathscr{M}_{2}$ ) is strongly convex since $H_{n}$ and $\mathscr{M}_{\varepsilon}$ are strongly convex and so $\lambda=b\left(\pi_{n}^{\prime}\right) \in$ $H_{n}+\mathscr{M}_{\varepsilon}$. Finally if we write $\lambda=\mu+\rho$ with $\mu \in H_{n}$ and $\|\rho\| \leq \varepsilon$, then $\nu=\alpha_{n} \mu \in H$, since $H$ is a cone, and $\left.\| b(\pi)-\nu\right] \| \leq 2 \varepsilon$. This shows that $b(\pi)$ is in the norm closure of $H$ and hence that $b(\pi) \in N$.

\section{Polarity}

5.1. Polarity operations. Given any sets $P \subset \mathscr{M}(E)$ and $J \subset \mathscr{K}(E)$ we define

$$
P^{0}=\{K \in \mathscr{K}(E): \mu(K)=0, \forall \mu \in P\}
$$

and

$$
J_{0}=\{\mu \in \mathscr{M}(E): \mu(K)=0, \forall K \in J\} .
$$

It is clear that $P^{0}$ is a $\sigma$-ideal in $\mathscr{K}$ that we shall call the polar $\sigma$-ideal of $P$ and $J_{0}$ is a band that we shall call the polar band of $J$.

5.2.a. Polar $\sigma$-ideals. A polar $\sigma$-ideal $I$ is a $\sigma$-ideal of the form $I=P^{0}$ for some $P \subset \mathscr{M}$; we shall then say that $I$ is defined by $P$. Of course such a set $P$ is not unique. However, it is easy to see that there is a largest family $M$ defining $I$, namely, $M=I_{0}$, to which we shall refer as the maximal band defining $I$. It follows from these simple remarks that a subset $I$ of $\mathscr{K}$ is a polar $\sigma$-ideal if and only if $I=\left(I_{0}\right)^{0}$.

It is clear that for any universally measurable set $A$ the $\sigma$-ideal $\mathscr{K}(A)$ is polar. In particular if $A$ is coanalytic then $\mathscr{K}(A)$ is an example of a coanalytic 
polar $\sigma$-ideal. Also as mentioned in the introduction the coanalytic $\sigma$-ideals $U_{0}$ and $\mathscr{K}_{\omega}(E)$ are both polar, however $U$ is not (see $\S 10$ ).

Inside the class of $\mathbf{G}_{\delta} \sigma$-ideals, the $\sigma$-ideal of compact sets of measure 0 (for some fixed measure) is polar. But on a perfect space $E$ the $\sigma$-ideal all of compact sets with empty interior is not polar, since its polar band is reduced to $\{0\}$. This also shows that $\mathscr{K}$ is the unique polar $\sigma$-ideal which contains all compact sets with empty interior.

5.2.b. Polar bands. A polar band $M$ is a band of the form $M=J_{0}$ for some $J \subset \mathscr{H}$. Notice that a polar band is necessarily a strong band. Also $I=M^{0}$ is the largest family in $\mathscr{K}$ defining $M$, and a subset $M$ of $\mathscr{M}$ is a polar band if and only if $M=\left(M^{0}\right)_{0}$.

It is also clear that for any universally measurable set $A$ the band $\mathscr{M}(A)$ is polar. Thus Proposition 3.1 gives examples of polar bands having different descriptive complexity. An interesting example of a polar band is given by $M_{0}(\mathrm{~T})$ : This result is due to R. Lyons (see [13]) but can also be derived from the next abstract theorem of Mokobodzki.

5.3. Bipolarity. Consider again arbitrary sets $P \subset \mathscr{M}$ and $J \subset \mathscr{K}$. One can try to describe $\left(P^{0}\right)_{0}$ and $\left(J_{0}\right)^{0}$-similarly to the biorthogonal theorem-as hulls of $P$ and $J$ for some properties. We do not know about any result concerning $\left(J_{0}\right)^{0}$; however, a complete description of $\left(P^{0}\right)_{0}$ is given by a theorem due to G. Mokobodzki when $P$ is analytic. Using our notation we can state Mokobodzki's Theorem as follows. For details see [3, p. 187] or [7, p. 313]; see also $\S 6.5$ of this paper.

Theorem (Mokobodzki). If $P$ is an analytic subset of $\mathscr{M}$, then $M=\left(P^{0}\right)_{0}$ is the strong band generated by $P$.

As consequences of this theorem we have:

(a) Let $M$ be an analytic subset of $\mathscr{M}$; then $M$ is a polar band iff $M$ is a strong band.

(b) If a polar $\sigma$-ideal $I$ is defined by some analytic family of measures, then the maximal band defining $I$ is also analytic.

(c) If $M$ is an analytic strong band, then the orthogonal band $M^{\prime}$ is generated by all measures $\mu$ such that the support of $\mu$ is in the polar $\sigma$-ideal $M^{0}$.

5.4. Polarity basis. Let $I$ be a $\sigma$-ideal in $\mathscr{K}$. We shall say that $B \subset I$ is a polarity basis or a $p$-basis for $I$ if any measure which is null on $B$ is null on $I$. Using the previous notation this means that $B_{0}=I_{0}$. Clearly any basis is a $p$-basis. Also as for a basis, given a $\sigma$-ideal it is natural to ask whether it has a simple $p$-basis. The following results can be proved by the same arguments as for a basis (see [8]).

Proposition 5.5. Let $I$ be a $\sigma$-ideal in $\mathscr{K}$.

(a) If I has, $\Sigma_{1}^{1}$ p-basis, then I has a Borel (and even $\mathbf{G}_{\delta}$ ) p-basis.

(b) If $I$ is $\Pi_{1}^{1}$ and has a Borel p-basis, then $I$ has a Borel p-basis which is an ideal.

However, in spite of this formal similarity between the properties of basis and $p$-basis, the following examples show that these notions are far from coinciding. 
Proposition 5.6. If $A$ is an analytic (Borel) subset of $E$, then the $\sigma$-ideal $\mathscr{K}(A)$ has an analytic (Borel) p-basis.

Proof. Suppose $A$ analytic and fix $\varphi: P \rightarrow A$ a continuous mapping from some Polish space $P$ onto $A$. Then the natural extension of $\varphi$ from $\mathscr{K}(P)$ to $\mathscr{K}(A)$ (which sends any compact $K \in \mathscr{K}(P)$ to $\varphi(K) \in \mathscr{K}(A)$ ) is also continuous, so the set $B=\{\varphi(K) ; K \in \mathscr{K}(P)\}$ is an analytic subset of $\mathscr{K}(A)$. And since any measure on $A$ is the image of some measure on $P$, it follows that $B$ is a $p$-basis for $\mathscr{K}(A)$.

If $A$ is Borel, then we can suppose moreover that $\varphi$ is one to one and so for its extension to $\mathscr{K}(P)$. It follows that $B$ is an injective continuous image of the Polish space $\mathscr{K}(P)$ and so $B$ is Borel.

Example 5.7. Let $E=X \times Y$ be the product space of an arbitrary uncountable compact metric space $X$ with $Y=[0,1]$. For any $A \subset E$ and any $x \in X$ let $A(x)=\{y \in Y:(x, y) \in A\}$. Then the $\sigma$-ideal:

$$
I=\{K \in \mathscr{K}(E): K(x) \text { is countable for all } x \in X\}
$$

is coanalytic and has a $\mathbf{G}_{\delta}$ hereditary $p$-basis, but has no Borel basis.

Proof. $I$ is clearly coanalytic. If $G$ denotes the set of all closed partial graphs from $X$ to $Y$ (that is, the set of all $K \in \mathscr{K}(E)$ such that $\operatorname{card}(K(x)) \leq 1$ for any $x \in X$ ), then $G$ is a hereditary $\mathbf{G}_{\delta}$ subset of $I$ and we shall prove that $G$ is a $p$-basis for $I$. To see this notice that if $\mu$ is a measure on $E$ and $K \in I$, then it follows from a classical result of Lusin that we can find a sequence $\left(K_{n}\right)_{n \in \omega}$ of closed partial graphs contained in $K$ and such that $\mu\left(K \backslash \bigcup_{n \in \omega} K_{n}\right)=0$; so if $\mu$ is null on $G$, then $\mu$ is null on $I$.

To prove that $I$ has no Borel basis we shall make use of the following result from [1]:

Theorem (Debs-Saint Raymond). Let I be a $\sigma$-ideal of $\mathscr{K}(E)$ satisfying.

(a) For any $L \in \mathscr{K} \backslash I$ and any sequence $\left(K_{n}\right)_{n \in \omega}$ in $I$ there exists $L^{\prime} \in$ $\mathscr{K} \backslash I$ such that $L^{\prime} \subset L \backslash \bigcup_{n \in \omega} K_{n}$.

(b) For any $L \in \mathscr{K} \backslash I$ the $\sigma$-ideal $I(L)=I \cap \mathscr{K}(L)$ is not Borel.

(c) I has a Borel basis.

If $A$ is an analytic subset of $E$ satisfying $\mathscr{K}(A) \subset I$, then there exists a sequence $\left(K_{n}\right)_{n \in \omega}$ in I such that $A \subset \bigcup_{n \in \omega} K_{n}$.

It is clear that the $\sigma$-ideal $I$ of 5.7 satisfies condition (a). Let $L \in \mathscr{K} \backslash I$ and $x_{0} \in E$ such that $L_{0}=L\left(x_{0}\right)$ is uncountable, and consider the mapping $\varphi: \mathscr{K}(L) \rightarrow \mathscr{K}\left(L_{0}\right)$ defined by $\varphi(K)=K\left(x_{0}\right)$. Then $\varphi(I \cap \mathscr{K}(L))$ is the $\sigma-$ ideal $\mathscr{K}_{\omega}\left(L_{0}\right)$ of all countable compact subsets of $L_{0}$. Since $L_{0}$ is uncountable, by a classical result of Hurewicz $\mathscr{K}_{\omega}\left(L_{0}\right)$ is not analytic; and since $\varphi$ is Borel, we conclude that $I \cap \mathscr{K}(L)$ is not analytic. So $I$ satisfies condition (b).

Now suppose by contradiction that $I$ satisfies also (c). Then it follows from the conclusion of the previous theorem that given any Borel function $f: X \rightarrow Y$ one can find a sequence $\left(f_{n}\right)_{n \in \omega}$ of closed partial graphs from $X$ to $Y$ such that $f \subset \bigcup_{n \in \omega} f_{n}$; moreover, since the functions $f_{n}: \operatorname{Dom}\left(f_{n}\right) \rightarrow Y$ are then continuous, one can even suppose, using Urysohn's Theorem, that $\operatorname{Dom}\left(f_{n}\right)=$ $X$.

To construct a Borel function $f: X \rightarrow Y$ contradicting the last statement, consider the space $\mathscr{C}(X, Y)$ of all continuous functions from $X$ to $Y$ endowed 
with the uniform convergence topology. Since $X$ is uncountable, we can find a Borel isomorphism $\Phi$ from $X$ onto the Polish space $\mathscr{C}(X, Y)^{\omega}$. Let $\left(\Phi_{n}\right)_{n \in \omega}$ denote the components of $\Phi$ (so for any $x \in X, \Phi_{n}(x): X \rightarrow Y$ is a continuous function). Then

$$
G=\left\{(x, y) \in X \times Y: \Phi_{n}(x) \neq y, \forall n \in \omega\right\}
$$

is a Borel subset of $X \times Y$ with co-countable $Y$-sections and hence admits a Borel selector $f$ (see, for example, [2]). Now given any sequence $\bar{f}=\left(f_{n}\right)_{n \in \omega} \in$ $\mathscr{C}(X, Y)^{\omega}$ if we let $a=\Phi^{-1}(\bar{f}) \in X$, then we have $f_{n}=\Phi_{n}(a)$ and in particular $f_{n}(a)=\Phi_{n}(a)(a)$ for all $n$. But by the selector property of $f$ we have also $f(a) \neq \Phi_{n}(a)(a)$ for all $n$. Hence $f(a) \neq f_{n}(a)$ for all $n$ and so $f$ is not contained in $\bigcup_{n \in \omega} f_{n}$.

\section{Polar $\sigma$-ideals with Borel $p$-Basis}

6.1. Standard bands. We shall say that $M$ is a standard band if $M$ is a Borel strong band.

The bands $\mathscr{M}_{c}(E), M_{0}(\mathrm{~T})$, and $\mathscr{M}(A)$ for $A$ Borel are standard bands. We recall that the first two examples have polar $\sigma$-ideals with Borel basis, whereas in the last example the $\sigma$-ideal $\mathscr{K}(A)$ may have no Borel basis (see 1.4(c)).

Theorem 6.2. If $M$ is a standard band then:

(a) The polar $\sigma$-ideal $M^{0}$ has a Borel p-basis.

(b) The orthogonal band $M^{\prime}$ is Borel.

The proof of this result which requires some preliminaries and will make large use of the Effective Descriptive Set Theory, will be given at the end of this section.

Corollary 6.3. Let $P$ be an analytic strongly convex subset of $\mathscr{M}$ and $M$ the band generated by $P$. Then the following conditions are equivalent:

(i) The band $M$ is Borel.

(ii) The polar $\sigma$-ideal $M^{0}$ has a Borel p-basis.

(iii) The orthogonal band $M^{\prime}$ is Borel.

Proof. Notice that by Theorem 4.4, $M$ is an analytic strong band.

It is easy to check that each of conditions (ii) and (iii) implies that $M$ is coanalytic and hence Borel. The other implications follow from Theorem 6.2.

Corollary 6.4. The family of standard bands is stable under countable intersections and countable sums.

Proof. For the countable intersection operation the stability is obvious since each of the notions of band, Borel set, and strongly convex set is stable by this operation.

If $\left(M_{n}\right)_{n \in \omega}$ is a sequence of standard bands, then $M=\sum_{n \in \omega} M_{n}$ is a priori an analytic strong band and we have clearly $M^{\prime}=\bigcap_{n \in \omega} M_{n}^{\prime}$ which is Borel since by Theorem 6.2 each $M_{n}^{\prime}$ is Borel, so $M=\left(M^{\prime}\right)^{\prime}$ is coanalytic (see 3.3) and hence Borel.

6.5. Mokobodzki's capacity $\gamma$. For the proof of the result we mentioned in 5.3 , G. Mokobodzki introduces for each fixed measure $\lambda$, a set function $\gamma$ 
(depending on $\lambda$ ) and defined on all subsets of $\mathscr{M}$, by setting for any $P \subset \mathscr{M}$ :

$$
\gamma(P)=\inf \{\sup \{\mu(f)+\lambda(1-f) ; \mu \in P\} ; f \in \mathscr{B}\}
$$

where $\mathscr{B}$ denotes the family of all Borel functions $f: E \rightarrow[0,1]$. The basic properties of $\gamma$ are the following:

(1) $\gamma$ is increasing.

(2) If $\left(Q_{n}\right)_{n \in \omega}$ is a decreasing sequence of compact sets, then $\gamma\left(\bigcap_{n \in \omega} Q_{n}\right)=$ $\inf _{n \in \omega} \gamma\left(Q_{n}\right)$.

(3) If $\left(P_{n}\right)_{n \in \omega}$ is an increasing sequence of arbitrary sets, then $\gamma\left(\bigcup_{n \in \omega} P_{n}\right)=$ $\sup _{n \in \omega} \gamma\left(P_{n}\right)$.

This means that $\gamma$ is a Choquet capacity. Now suppose that $Q$ is a compact convex subset of $\mathscr{M}$; then by the Min-Max Theorem we can switch the inf and the sup in the formula defining $\gamma(Q)$ and obtain that for such $Q$

$$
\gamma(Q)=\sup \{\|\mu \wedge \lambda\| ; \mu \in Q\} .
$$

Finally applying the Choquet capacitability theorem one extends the last formula for any subset $Q$ of $\mathscr{M}$ which is analytic and strongly convex.

6.6. An effective version of $\gamma$. Now we suppose that the compact space $E$ is recursively presentable. Given any fixed measure $\lambda$ on $E$, we introduce a set function $\gamma_{0}$ (depending on $\lambda$ ) and defined on all subsets of $\mathscr{M}$, by setting for any $P \subset \mathscr{M}$ :

$$
\gamma_{0}(P)=\inf \left\{\sup \{\mu(f)+\lambda(1-f) ; \mu \in P\} ; f \in \mathscr{B}_{0}\right\}
$$

where $\mathscr{B}_{0}$ denotes the (countable) family of all functions $f: E \rightarrow[0,1]$ which are $\Delta_{1}^{1}(\lambda)$. This natural variation of $\gamma$ is considered in [9, Section 2] as an example of an effective capacity. The basic properties of $\gamma_{0}$ are the following:

(1) $\gamma_{0}$ is increasing.

(2) If $\left(Q_{n}\right)_{n \in \omega}$ is a decreasing sequence of compact sets, then $\gamma_{0}\left(\bigcap_{n \in \omega} Q_{n}\right)=$ $\inf _{n \in \omega} \gamma_{0}\left(Q_{n}\right)$.

(3) If $\left(P_{n}\right)_{n \in \omega}$ is an increasing $\Sigma_{1}^{1}(\lambda)$, sequence, then $\gamma_{0}\left(\bigcup_{n \in \omega} P_{n}\right)=$ $\sup _{n \in \omega} \gamma_{0}\left(P_{n}\right)$.

Property (1) is obvious. Property (2) follows from the fact that $\gamma_{0}$ coincides with $\gamma$ on all compact sets [9, Lemma 2.13]. Property (3) is stated in [9, Lemma 2.14] only for $\Delta_{1}^{1}$-sequences assuming $\lambda$ is $\Delta_{1}^{1}$, but the proof is clearly uniform in $\lambda$ and is still valid for $\Sigma_{1}^{1}$-sequences.

Then similarly to $\gamma$ one can deduce from this and the Min-Max Theorem that for any $\Sigma_{1}^{1}(\lambda)$ strongly convex set $Q \subset \mathscr{M}$

$$
\gamma_{0}(Q)=\sup \{\|\mu \wedge \lambda\| ; \mu \in Q\} \text {. }
$$

Proof of Theorem 6.2. Let $M$ be a Borel strong band. Since the arguments of the proof are uniform, we can suppose that $M$ is $\Delta_{1}^{1}$ in some recursive presentation of $E$.

(a) For any $\lambda \in M^{c}$ we have

$$
\sup \{\|\mu\| ; \mu \in M, \mu \leq \lambda\}=\sup \{\|\mu \wedge \lambda\| ; \mu \in M\}<\|\lambda\| \text {. }
$$

Since $M$ is $\Delta_{1}^{1}$ and strongly convex, it follows from the formula of the last paragraph that there exists $f: E \rightarrow[0,1]$ which is $\Delta_{1}^{1}(\lambda)$ and such that

$$
\sup \{\mu(f)+\lambda(1-f) ; \mu \in M\}<\|\lambda\| \text {. }
$$


Hence by the homogeneity of $M$ we have

$$
\left\{\begin{array}{l}
\lambda(f)>0, \\
\forall \mu \in M, \mu(f)=0 .
\end{array}\right.
$$

Then the set $S=\left\{f \geq \frac{\lambda(f)}{\|\lambda\|}\right\}$ is $\Delta_{1}^{1}(\lambda)$ and $\lambda(S)>0$, so we can find a $\Delta_{1}^{1}(\lambda)$ compact set $K \subset S$ such that $\lambda(K)>0$. But since $K \subset S$ we have also that $\mu(K)=0$ for any $\mu \in M$ and hence $K \in M^{0}$.

So for any $\lambda \in M^{c}$ we can find a $\Delta_{1}^{1}(\lambda)$ compact set $K \in M^{0}$ such that $\lambda(K)>0$. But $M^{0}$ is $\Pi_{1}^{1}$ (since $M$ is $\Delta_{1}^{1}$ ) and so by $\Delta_{1}^{1}$-choice we can find a $\Delta_{1}^{1}$ mapping $\Phi: M^{c} \rightarrow M^{0}$ satisfying $\lambda(\Phi(\lambda))>0$ for any $\lambda \in M^{c}$. Then the $\Sigma_{1}^{1}$ set $R=\Phi\left(M^{c}\right)$ is obviously a $\Sigma_{1}^{1} p$-basis for $M^{0}$. The conclusion follows then from (5.5).

(b) From $(5.3(\mathrm{c}))$ we have

$$
\begin{aligned}
\lambda \in M^{\prime} & \Leftrightarrow \forall \varepsilon>0, \exists K \in M^{0}: \lambda(K)>\|\lambda\|-\varepsilon \\
& \Leftrightarrow \forall \varepsilon>0, \exists K \in R: \lambda(K)>\|\lambda\|-\varepsilon,
\end{aligned}
$$

so $M^{\prime}$ is $\Sigma_{1}^{1}$ and, since $M^{\prime}$ is $\Pi_{1}^{1}$ as the orthogonal band of the $\Sigma_{1}^{1}$ band $M$, $M^{\prime}$ is $\Delta_{1}^{1}$.

\section{BOREL POLAR $\sigma$-IDEALS}

Proposition 7.1. Let $H$ be a compact convex subset of $\mathscr{M}$, and let $M$ be the band generated by $H$. Then:

(a) $M$ is a $\mathbf{K}_{\sigma \delta}$ strong band.

(b) The orthogonal band $M^{\prime}$ is $\mathbf{G}_{\delta}$.

(c) The polar $\sigma$-ideal $I=M^{0}$ is $\mathbf{G}_{\delta}$. Moreover, $I=\bigcap_{n \in \omega} J_{n}$ where each $J_{n}$ satisfies:

(1) $J_{n}$ is a hereditary open set in $\mathscr{K}$.

(2) If $K \in J_{n}$ and $L \in I$, then $K \cup L \in J_{n}$.

Proof. (a) $M$ is a strong band by Theorem 4.4, and if $N$ is the hereditary convex cone generated by $H$; then $N$ is $\mathbf{K}_{\sigma}$ and it follows from (2.4) that $M$ is $\mathbf{K}_{\sigma \delta}$.

(b) Since $M^{\prime}=H^{\prime}$, we have

$$
\lambda \notin M^{\prime} \Leftrightarrow \exists \mu \in H, \quad \mu \neq 0, \mu \leq \lambda
$$

which shows that $\mathscr{M} \backslash M^{\prime}$ is $\mathbf{K}_{\sigma}$.

(c) Consider $\gamma: \mathscr{K}(E) \rightarrow \mathbb{R}_{+}$defined by $\gamma(K)=\sup \{\mu(K) ; \mu \in H\}$. Then it follows from the Min-Max theorem that $\gamma$ is upper semicontinuous on $\mathscr{K}$. Since $\gamma$ is increasing and subadditive, it is clear that the sets $J_{n}=\{K \in$ $\left.\mathscr{K}: \gamma(K)<2^{-n}\right\}$ satisfy (1) and (2).

7.2. Elementary polar $\sigma$-ideals. A polar $\sigma$-ideal $I$ of the form $I=H^{0}$ for some compact convex set $H \subset \mathscr{M}$ will be said to be elementary; such a $\sigma$-ideal is $\mathbf{G}_{\delta}$. In fact, all examples of $\mathbf{G}_{\delta}$ polar $\sigma$-ideals, of which we know are of this form.

Notice that the set function $\gamma$ of the previous proof can be extended to all subsets of $E$ by $\gamma(A)=\sup \left\{\mu^{*}(A) ; \mu \in H\right\}$ and this extension defines a capacity on $E$. Such capacities are called normal capacities. It follows from these remarks that $I$ is an elementary polar $\sigma$-ideal iff $I$ is the $\sigma$-ideal of null sets for some normal capacity. 
Theorem 7.3. Let $M$ be an analytic strong band. Then the following conditions are equivalent:

(i) The polar $\sigma$-ideal $M^{0}$ is $\mathbf{G}_{\delta}$.

(ii) The polar $\sigma$-ideal $M^{0}$ has a p-basis which is a $\mathbf{G}_{\delta}$ is ideal.

(iii) The orthogonal band $M^{\prime}$ is $\mathbf{G}_{\delta}$.

Proof. (i) $\Rightarrow$ (ii) Obvious.

(ii) $\Rightarrow$ (iii) Let $J$ be a $\mathbf{G}_{\delta}$ ideal which is a $p$-basis for $M^{0}$. Since $J$ is hereditary, we can write $J=\bigcap_{n \in \omega} J_{n}$ where each $J_{n}$ is open and hereditary in $\mathscr{K}$. Let $\mathscr{W}_{n}$ be the set of all open sets $W$ such that $\bar{W} \in J_{n}$. We shall show that

$$
M^{\prime}=\bigcap_{\varepsilon>0} \bigcap_{n \in \omega} \bigcup_{W \in \mathscr{W}_{n}}\left\{\mu \in \mathscr{M}: \mu\left(W^{c}\right)<\varepsilon\right\}
$$

from which will show that $M^{\prime}$ is $\mathbf{G}_{\delta}$.

Denote by $M^{\prime \prime}$ the set defined by the right side of this equality. Suppose $\mu \in M^{\prime \prime}$; fix $\varepsilon>0$; and for any $n \in \omega$ fix $K_{n}=\bar{W}_{n} \in J_{n}$ satisfying $\mu\left(W_{n}^{c}\right)<$ $\frac{\varepsilon}{2^{n}}$. Then $K=\bigcap_{n \in \omega} K_{n} \in J$ and

$$
\mu\left(K^{c}\right) \leq \sum_{n=0}^{\infty} \mu\left(K_{n}^{c}\right) \leq \sum_{n=0}^{\infty} \mu\left(W_{n}^{c}\right)<2 \varepsilon .
$$

This shows that $\mu \in M^{\prime}$.

Conversely suppose $\mu \in M^{\prime}$; then from (5.3(c)) it follows that $\mu$ is carried by $\bigcup_{n \in \omega} L_{n}$ with $L_{n} \in M^{0}$. Since $J$ is a $p$-basis for $M^{0}$, we can take all the $L_{n}$ in $J$; and since $J$ is an ideal, we can assume that the sequence $\left(L_{n}\right)_{n \in \omega}$ is increasing. From these remarks one easily deduces that $\mu \in M^{\prime \prime}$.

(iii) $\Rightarrow$ (i) Observe that for any $K \in \mathscr{K}$ we have

$$
K \in \mathscr{K} \backslash M^{0} \Leftrightarrow \exists \mu \in \mathscr{M} \backslash M^{\prime}: \mu(K)=\mu(E) .
$$

Since $\mathscr{M} \backslash M^{\prime}$ is $\mathbf{K}_{\sigma}$, it follows that $\mathscr{K} \backslash M^{0}$ is $\mathbf{K}_{\sigma}$.

It follows from the proof of the Theorem 7.3 that the implication (iii) $\Rightarrow$ (i) holds for any subset $M$ of $\mathscr{M}$.

Let $M$ be a standard band with Borel polar $\sigma$-ideal $I=M^{0}$; then by Theorem 7.3 the band $M^{\prime}$ is $\mathbf{G}_{\delta}$. If moreover $I$ is elementary, then $M$ is $\mathbf{K}_{\sigma \delta}$. But in the general case we do not know if there is any bound for the Borel class of $M$. However, we have the following partial result:

Theorem 7.4. Let $M$ be a standard band and suppose that the polar $\sigma$-ideal $M^{0}$ has a p-basis $J$ of the form $J=\bigcap_{n \in \omega} J_{n}$ where each $J_{n}$ satisfies:

(1) $J_{n}$ is a hereditary open set in $\mathscr{K}(E)$.

(2) If $K \in J$ and $L \in J_{n}$, then $K \cup L \in J_{n}$.

Then $M$ is $\mathbf{K}_{\sigma \delta}$.

Proof. The conclusion follows from the next lemma.

We recall the notation $\mathscr{M}_{\varepsilon}=\{\mu \in \mathscr{M}:\|\mu\| \leq \varepsilon\}$.

Lemma 7.5. Under the hypothesis of Theorem 7.4, for any $\varepsilon>0$ there exists a $\mathbf{K}_{\sigma}$ set $H \subset \mathscr{M}$ such that $M \subset H \subset M+\mathscr{M}_{\varepsilon}$.

Proof. Fix $\varepsilon>0$ and $\varphi: P \rightarrow M$ continuous from some polish space $P$ onto $M$ and let

$$
P_{0}=\bigcup\left\{0 \text { open } \subset P: \exists H \in \mathbf{K}_{\sigma}(\mathscr{M}), \varphi(0) \subset H \subset M+\mathscr{M}_{\varepsilon}\right\} .
$$


Then there exists $H_{0}$ a $\mathbf{K}_{\sigma}$ set such that $\varphi\left(P_{0}\right) \subset H_{0} \subset M+\mathscr{M}_{\varepsilon}$. If $P=P_{0}$, then, since $M=\varphi(P)$, the conclusion of the lemma holds.

Suppose by contradiction that $P \neq P_{0}$, and let $Q=P \backslash P_{0}$. Then for any nonempty open subset $U$ of $Q$, we have $\overline{\varphi(U)} \not \subset M+\mathscr{M}_{\varepsilon}$ : otherwise, if $U=0 \cap Q$ with 0 open $\subset P$ we would have $\varphi(0) \subset \overline{\varphi(U)} \cup H_{0} \subset M+\mathscr{M}_{\varepsilon}$, hence $0 \subset P_{0}$ and $U=\varnothing$.

We suppose $J_{0}=\mathscr{K}(E)$. We shall construct by induction on $n$, open sets $U_{n}$ in $Q$ and open sets $W_{n}$ in $E$ satisfying:

(o) $U_{0}=Q, W_{0}=E$;

and for $n>0$ :

(i) $U_{n} \neq \varnothing, \bar{U}_{n} \subset U_{n-1}, \operatorname{diam} U_{n}<\frac{1}{n}$;

(ii) $)_{n} \lambda\left(W_{n}\right)>\varepsilon, \forall \lambda \in \varphi\left(U_{n}\right)$;

(iii) $\bigcup_{j=k}^{n}, W_{j} \in J_{k}, \forall k \leq n$.

Suppose $\left\langle U_{k}, W_{k}\right\rangle_{k \leq n}$ is constructed, and let $U$ be any nonempty open subset of $Q$ satisfying: $\bar{U} \subset U_{n}$ and $\operatorname{diam}(U)<\frac{1}{n+1}$. Fix some $\lambda_{0} \in \overline{\varphi(U)}$ such that $\lambda_{0} \notin M+\mathscr{M}_{\varepsilon}$. Then the $M^{\prime}$-part of $\lambda_{0}$ has a mass $>\varepsilon$, and by Mokobodzki's Theorem we can find $L \in J$ such that $\lambda_{0}(L)>\varepsilon$ (since $J$ is an ideal and a $p$-basis for $\left.M^{0}\right)$. Now from (iii) ${ }_{n}$ and the properties of $J_{n}$ we have that $L \cup \bigcup_{j=k}^{n} \bar{W}_{j} \in J_{k}$ for any $k \leq n$; and since all the $J_{k}$ are open and hereditary, we can find an open neighborhood $W$ of $L$ such that $\bar{W} \cup \bigcup_{j=k}^{n} \bar{W}_{j} \in J_{k}$ for any $k \leq n$. Then $\lambda_{0} \in \overline{\varphi(U)} \cap\{\lambda: \lambda(W)>\varepsilon\}$ and hence the open set $U^{\prime}=$ $U \cap \varphi^{-1}(\{\lambda: \lambda(W)>\varepsilon\})$ is nonempty. Finally it is clear that $U_{n+1}=U^{\prime}$ and $W_{n+1}=W$ satisfy $(\mathrm{i})_{n+1},(\mathrm{ii})_{n+1}$, (iii) $)_{n+1}$, and this finishes the construction.

By $(\mathrm{i})_{n}$ there exists $\alpha \in P$ such that $\{\alpha\}=\bigcap_{n \in \omega} U_{n}$, and so $\mu=\varphi(\alpha) \in$ $M \cap \varphi\left(U_{n}\right)$ for all $n$. Now consider the $\mathbf{G}_{\delta}$ set $A=\bigcap_{k \in \omega} \bigcup_{n \geq k} W_{n}$; it follows from $(\mathrm{ii})_{n}$ that

$$
\mu(A)=\mu\left(\lim _{n} \sup W_{n}\right) \geq \lim \sup \mu\left(W_{n}\right) \geq \varepsilon .
$$

But for any compact subset $K$ of $A$ we have

$$
\forall k \in \omega, \quad K \subset \bigcup_{j \geq k} W_{j}
$$

hence by compactness

$$
\forall k \in \omega, \quad \exists m_{k} \geq k: \quad K \subset \bigcup_{j=k}^{m_{k}} \bar{W}_{j},
$$

so by (iii) $n$ and since $J_{k}$ is hereditary, we have that $K \in J_{k}$ for any $k \in \omega$ and thus $K \in J$ and $\mu(K)=0$. It follows that $\mu(A)=0$, which gives the contradiction.

\section{Polar $\sigma$-ideals with Borel basis}

8.1. Intersection of $\sigma$-ideals. Let $I_{1}$ and $I_{2}$ be two $\sigma$-ideals and $I=I_{1} \cap I_{2}$. (a) If $B_{i}$ is a basis for $I_{i}$, for $i=1,2$, then $B=\left\{K_{1} \cap K_{2} ; K_{1} \in B_{1}, K_{2} \in\right.$ $B_{2}$ \} is a basis for $I$.

(b) If $I_{1}$ and $I_{2}$ have analytic basis, then the same holds for $I$.

(c) If $I_{1}$ and $I_{2}$ are coanalytic with Borel basis then the same holds for $I$. 
The verification of (a) is straightforward. Since the mapping $\left(K_{1}, K_{2}\right) \mapsto$ $K_{1} \cap K_{2}$ is Borel from $\mathscr{K} \times \mathscr{K}$ into $\mathscr{K},(b)$ follows from (a). Since for a coanalytic $\sigma$-ideal the existence of a Borel basis is equivalent to the existence of an analytic basis, (c) follows from (b). As an immediate consequence we have

Proposition 8.2. Let $M_{1}$ and $M_{2}$ be two analytic bands and $M=M_{1}+M_{2}$. If the polar $\sigma$-ideals $M_{1}^{0}$ and $M_{2}^{0}$ have Borel basis, then $M^{0}$ has a Borel basis.

Let $I$ be a $\sigma$-ideal. A nonempty compact set $K \in E$ will be said to be $I$-perfect if for any nonempty relatively open set $V$ in $K$ we have $\bar{V} \notin I$. The family $I_{p}$ of $I$-perfect sets is exactly the complement of what is denoted by $I^{\text {loc }}$ in [8].

Suppose the $\sigma$-ideal $I$ coanalytic. Then it is clear that $I_{p}$ is analytic and by a result of Kechris-Louveau-Woodin [8] $I_{p}$ is Borel if and only if $I$ has a Borel basis.

Lemma 8.3. Let $M$ be a band. Then for a nonempty compact set $K \subset E$, the following conditions are equivalent:

(i) $K$ is $M^{0}$-perfect.

(ii) $M(K)=M \cap \mathscr{M}(K)$ is dense in $\mathscr{M}(K)$.

(iii) $K$ is the support of some measure $\mu \in M$.

Proof. (i) $\Rightarrow$ (ii) Since $M(K)$ is a convex cone, it is sufficient to prove that any Dirac measure on $K$ is in the closure of $M(K)$. So fix $x \in K$ and $\left(V_{n}\right)_{n \in \omega}$ a basis of neighborhoods of $x$ in $K$. Since $K$ is perfect, for any $n \in \omega$ there exists $\mu_{n} \in M$ such that $\mu_{n}\left(\bar{V}_{n}\right)>0$, then $\lambda_{n}=\left.\frac{1}{\mu_{n}\left(\bar{V}_{n}\right)} \mu_{n}\right|_{\bar{V}_{n}} \in M(K)$ and $\lim _{n \rightarrow \infty} \lambda=\delta_{x}$.

(ii) $\Rightarrow$ (iii) Fix an enumeration of a basis $\left(W_{n}\right)$ for nonempty open sets in $K$. For any $n \in \omega$ choose $\mu_{n}$ in $M(K) \cap\left\{\mu \in \mathscr{M}(K): \mu\left(W_{n}\right)>0\right\}$, then the measure $\mu=\sum_{n=0}^{\infty} \frac{1}{2^{n}\left\|\mu_{n}\right\|} \mu_{n}$ satisfies (iii).

(iii) $\Rightarrow$ (i) immediate.

Proposition 8.4. If $M$ is a $\mathbf{G}_{\delta}$ band, then the polar $\sigma$-ideal $M^{0}$ has a Borel basis.

Proof. (a) Let $M=\bigcap_{n \in \omega} G_{n}$ with each $G_{n}$ open in $\mathscr{M}$; then by condition (ii) of the previous lemma and the Baire Theorem, $K$ is $M^{0}$-perfect if and only if $G_{n} \cap \mathscr{M}(K)$ is dense in $\mathscr{M}(K)$ for any $n \in \omega$. Then a simple complexity computation shows that $M_{p}^{0}$ is $\mathbf{K}_{\sigma \delta}$.

From the last proposition, Theorem 4.4, and Corollary 6.3 we obtain

Corollary 8.5. The strong band generated by $a \mathbf{G}_{\delta}$ band is Borel.

\section{FUNCTIONAL REPRESENTATION OF STANDARD BANDS}

Let $M$ be a band of the form

$$
M=\left\{\mu \in \mathscr{M}: \lim _{\mathscr{S}} \mu\left(f_{n}\right)=0\right\}
$$

where $\left(f_{n}\right)_{n \in \omega}$ is a sequence of continuous functions on $E$ with $0 \leq f_{n} \leq 1$ and $\mathscr{F}$ a filter on $\omega$. 
If $\mathscr{F}$-viewed as a subset of the compact metric space $\mathscr{P}(\omega) \approx\{0,1\}^{\omega}$-is Borel, then the band $M$ is also Borel in $\mathscr{M}$. Now suppose that $\mathscr{F}$ satisfies the "dominated convergence theorem" in the sense that "for any probability space $(\Omega, \Sigma, P)$ and any uniformly bounded sequence $\left(\varphi_{n}\right)$ of measurable functions $\varphi_{n}: \Omega \rightarrow \mathbb{R}$ such that $\varphi(t)=\lim _{\mathscr{F}} \varphi_{n}(t)$ exists a.e., we have $\int \varphi(t) d P(t)=$ $\lim _{\mathscr{F}} \int \varphi_{n}(t) d P(t)$ "; then under this last assumption on $\mathscr{F}$ it is easy to check that $M$ is strongly convex.

So if $\mathscr{F}$ is a Borel filter satisfying the "dominated convergence theorem", then $M$ is a standard band. The aim of this section is to prove that any standard band is of this form. Notice that in general if $M$ is a band of this form there is no easy description of the orthogonal band $M^{\prime}$ of the same type. However, the representation we shall give here for a standard band will also give a very simple description of $M^{\prime}$.

For the proof of the main result we will follow a method used by A. Louveau in [10] to give a representation for all Borel and strongly affine functions on a compact convex set, using the Fréchet iterated filters.

9.1. Fréchet iterated filters. The filters we consider here can be defined in several natural ways. For an elegant presentation and for more details we refer to [10]. We only state here properties that we shall use in the sequel.

Fix some bijection $(n, k) \mapsto\langle n, k\rangle$ from $\omega \times \omega$ onto $\omega$. Then there exists on $\omega$ an increasing family $\left(\mathscr{F}_{\xi}\right)_{\xi<\omega_{1}}$ of filters, starting from $\mathscr{F}_{0}$ the Fréchet filter and satisfying for any $\xi<\omega_{1}$ :

(a) $\mathscr{F}_{\xi}$ is a Borel filter.

(b) $\mathscr{F}_{\xi}$ satisfies the "dominated convergence theorem".

(c) For any double sequence $\left(\alpha_{n, k}\right)$ in $\mathbb{R}$, we have

$$
\varlimsup_{\mathscr{S}_{0}}\left(\varlimsup_{\mathscr{F}_{\xi}}\left(\alpha_{n, k}\right)_{k}\right)_{n}=\varlimsup_{\mathscr{F}_{\xi+1}} \alpha_{\langle n, k\rangle} .
$$

Theorem 9.2. If $M$ is a standard band, then there exists a sequence $\left(f_{n}\right)_{n \in \omega}$ of continuous functions on $E$ with $0 \leq f_{n} \leq 1$ and a countable ordinal $\xi$ such that

$$
M=\left\{\mu \in \mathscr{M}: \lim _{\mathscr{F}_{\xi}} \mu\left(f_{n}\right)=0\right\} \quad \text { and } \quad M^{\prime}=\left\{\mu \in \mathscr{M}: \varlimsup_{\mathscr{F}_{\xi}} \mu\left(f_{n}\right)=\mu(1)\right\} .
$$

Proof. Denote by $\mathscr{C}$ the set of all continuous functions $f: E \rightarrow[0,1]$. For any $\varepsilon>0$ we define

$$
\Gamma_{\varepsilon}: \mathscr{P}(\mathscr{M}) \times \mathscr{P}(\mathscr{M}) \rightarrow\{0,1\}
$$

by

$$
\begin{aligned}
\Gamma_{\varepsilon}(A, B) & =0 \text { if and only if : } \\
\exists\left(f_{n}\right)_{n \in \omega} \subset \mathscr{C}, \quad \exists \xi<\omega_{1}: & \left\{\begin{array}{l}
\forall \mu \in A, \quad \overline{\lim }_{\mathscr{F}_{\xi}} \mu\left(f_{n}\right) \leq \varepsilon \\
\text { and } \\
\forall \nu \in B, \quad \overline{\lim }_{\mathscr{F}_{\xi}} \nu\left(f_{n}\right) \geq \nu(1)-\varepsilon .
\end{array}\right.
\end{aligned}
$$

If $\Gamma_{\varepsilon}(A, B)=0$ we shall call $\xi$-test for $(A, B)$ any sequence $\left(f_{n}\right)$ satisfying the previous properties. $\Gamma_{\varepsilon}$ satisfies the following properties:

(1) $\Gamma_{\varepsilon}$ is monotone. 
(2) If $\left(A_{n}\right)_{n \in \omega}$ and $\left(B_{n}\right)_{n \in \omega}$ are increasing sequences in $\mathscr{P}(\mathscr{M})$ with $A=$ $\bigcup_{n \in \omega} A_{n}$ and $B=\bigcup_{n \in \omega} B_{n}$, then $\Gamma_{\varepsilon}(A, B)=\sup _{n \in \omega} \Gamma_{\varepsilon}\left(A_{n}, B_{n}\right)$.

(3) If $A$ and $B$ are two analytic sets in $\mathscr{P}(\mathscr{M})$ with $\Gamma_{\varepsilon}(A, B)=1$, then there exist two decreasing sequences $\left(H_{n}\right)_{n \in \omega}$ and $\left(K_{n}\right)_{n \in \omega}$ of compacts sets in $\mathscr{P}(\mathscr{M})$ such that

(i) $\Gamma_{\varepsilon}\left(H_{n}, K_{n}\right)=1, \forall n \in \omega$;

(ii) $\bigcap_{n \in \omega} H_{n} \subset A$ and $\bigcap_{n \in \omega} K_{n} \subset B$.

(1) is obvious. To prove (2) it is sufficient to prove that if $\Gamma_{\varepsilon}\left(A_{n}, B_{n}\right)=0$ for any $n$, then $\Gamma_{\varepsilon}(A, B)=0$. To see this notice that if $\left(f_{n, k}\right)_{k \in \omega}$ is a $\xi$-test for $\left(A_{n}, B_{n}\right)$, then by $9.1(\mathrm{c})$ the $\omega$-sequence $\left(f_{\langle n, k\rangle}\right)$ is a $(\xi+1)$-test for $(A, B)$. Finally (3) is just a variation of the Choquet capacitability theorem as used also in [10].

Now consider a standard band $M$ and let $M^{\prime}$ be its orthogonal band. We claim that $\Gamma_{\varepsilon}\left(M, M^{\prime}\right)=0$. To see this notice first that by Theorem 6.2 , both $M$ and $M^{\prime}$ are analytic (in fact Borel). Suppose by contradiction that $\Gamma_{\varepsilon}\left(M, M^{\prime}\right)=1$. Consider $\left(H_{n}\right)_{n \in \omega}$ and $\left(K_{n}\right)_{n \in \omega}$ given by (3) for $(A, B)=\left(M, M^{\prime}\right)$, and let $H=\bigcap_{n \in \omega} H_{n}$ and $K=\bigcap_{n \in \omega} K_{n}$. Since $M$ is strongly convex, we have conv $H \subset M$ and so for any $\nu \in K$ we can find (see $[7$, p. 317, Lemma 6]) a continuous function $g: E \rightarrow[0,1]$ such that $\mu(g)+\nu(1-g)<\varepsilon$ for any $\mu \in H$, so $\nu(g)>\nu(1)-\varepsilon$ and $H \subset\{\mu: \mu(g)<\varepsilon\}$. Then by compactness we can find a finite sequence $\left(g_{i}\right)_{1 \leq i \leq m}$ in $\mathscr{E}$ such that

$$
H \subset V=\bigcap_{i=1}^{m}\left\{\mu: \mu\left(g_{i}\right)<\varepsilon\right\} \text { and } K \subset W=\bigcup_{i=1}^{m}\left\{\nu: \nu\left(g_{i}\right)>\nu(1)-\varepsilon\right\} .
$$

Hence there exists some index $n$ such that $H_{n} \subset V$ and $K_{n} \subset W$. Now consider the infinite sequence $\left(g_{k}\right)_{k \in \omega}$ obtained by repeating the finite sequence $\left(g_{i}\right)_{1 \leq i \leq m} ;$ then

$$
\left\{\begin{array}{l}
\forall \mu \in H_{n}, \quad \overline{\lim }_{k} \mu\left(g_{k}\right) \leq \varepsilon \\
\text { and } \\
\forall \nu \in K_{n}, \quad \overline{\lim }_{k} \nu\left(g_{k}\right) \geq \nu(1)-\varepsilon .
\end{array}\right.
$$

Since $\mathscr{F}_{0}$ is the Fréchet filter, $\left(g_{k}\right)_{k \in \omega}$ is a 0 -test sequence for $\left(H_{n}, K_{n}\right)$ and $\Gamma_{\varepsilon}\left(H_{n}, K_{n}\right)=0$, which is a contradiction and proves that $\Gamma_{\varepsilon}\left(M, M^{\prime}\right)=0$.

Now applying this with $\varepsilon=\frac{1}{p+1}$ we obtain for any $p \in \omega$, a sequence $\left(g_{p, k}\right)_{k}$ in $\mathscr{C}$ satisfying for some $\xi$ :

$$
\left\{\begin{array}{l}
\forall p \in \omega, \forall \mu \in M, \quad \varlimsup_{\lim _{\mathcal{F}_{\xi}}} \mu\left(g_{p, k}\right)_{k} \leq \frac{1}{p+1} \\
\text { and } \\
\forall p \in \omega, \forall \nu \in M^{\prime}, \quad \varlimsup_{\mathcal{F}_{\xi}} \nu\left(g_{p, k}\right)_{k} \geq \nu(1)-\frac{1}{p+1} .
\end{array}\right.
$$

Let $\mathscr{F}=\mathscr{F}_{\xi+1}$ and denote by $\left(f_{n}\right)$ the sequence $\left(g_{\langle p, k\rangle}\right)$; then by $(9.1(\mathrm{c}))$ we have

$$
\begin{cases}\forall \mu \in M, & \overline{\lim }_{\mathscr{F}} \mu\left(f_{n}\right)=0 \\ \text { and } & \\ \forall \nu \in M^{\prime}, & \varlimsup_{\mathscr{I}} \nu\left(f_{n}\right)=\nu(1)\end{cases}
$$

Thus

$$
M \subset\left\{\mu: \lim _{\mathscr{F}} \mu\left(f_{n}\right)=0\right\} \quad \text { and } \quad M^{\prime} \subset\left\{\nu: \varlimsup_{\mathscr{S}} \nu\left(f_{n}\right)=\nu(1)\right\} .
$$


In fact, both inclusions are equalities. To see this, consider any $\lambda \in \mathscr{M}$ and let $\lambda=\mu+\nu$ with $\mu \in M$ and $\nu \in M^{\prime}$, then

$$
\varlimsup_{\mathscr{F}} \nu(f) \leq \varlimsup_{\mathscr{F}} \lambda\left(f_{n}\right) \leq \varlimsup_{\mathscr{F}} \mu\left(f_{n}\right)+\varlimsup_{\mathscr{F}} \nu\left(f_{n}\right) .
$$

And since $\lim _{\mathscr{F}} \mu\left(f_{n}\right)=0$ and $\lim _{\mathscr{F}} \nu\left(f_{n}\right)=\nu(1)$, we conclude that

$$
\varlimsup_{\mathscr{F}} \lambda\left(f_{n}\right)=\nu(1) \text {. }
$$

In particular,

$$
\lambda \in M \Leftrightarrow \lim _{\mathscr{F}} \lambda\left(f_{n}\right)=0
$$

and

$$
\lambda \in M^{\prime} \Leftrightarrow \varlimsup_{\mathscr{F}} \lambda\left(f_{n}\right)=\lambda(1) .
$$

\section{Problems}

1. Construct a Borel band $M$ such that the orthogonal band $M^{\prime}$ is not Borel. In view of the remark following Theorem 7.3, if $I$ is a $\mathbf{G}_{\delta} \sigma$-ideal such that the polar band $N$ is not Borel, then the $\mathbf{G}_{\delta}$ band $M=N^{\prime}$ would do.

2. Construct Borel bands $M_{1}$ and $M_{2}$ such that the band $M_{1}+M_{2}$ is not Borel. Notice that for such bands, since $M_{1}+M_{2}=\left(M_{1}^{\prime} \cap M_{2}^{\prime}\right)^{\prime}$, the band $M_{1}^{\prime} \cap M_{2}^{\prime}$ is not Borel; it follows that one of the bands $M_{1}$ or $M_{2}$ would answer Question 1 positively.

3. Is there any structural result on $\mathbf{G}_{\delta}$ bands or $\mathbf{G}_{\delta}$ strong bands, which for example would "explain" Corollary 8.5?

4. Let $M$ be a standard band so that both bands $M$ and $M^{\prime}$ are Borel. Is there any general relation between the Borel classes of $M$ and $M^{\prime}$ ? In particular, if $M$ is a standard band such that $M^{\prime}$ is $\mathbf{G}_{\delta}$, is $M$ necessarily of class $\mathbf{K}_{\sigma \delta}$ ?

5. Is any $\mathbf{G}_{\delta}$ polar $\sigma$-ideal elementary in the sense of 7.2?

6. Given some family $J$ of compact sets, is there any description of the maximal $\sigma$-ideal $\left(J_{0}\right)^{0}$ in the same spirit of Mokobodzki's Theorem?

We finish this section by discussing some questions concerning the $\sigma$-ideals $U$ and $U_{0}$ considered in the introduction.

7. We recall that $U_{0}$ is a polar $\sigma$-ideal. In a first version of this work we asked whether the same holds for $U$. The following negative answer was kindly communicated to us by the referee. The arguments are based on the remarkable constructions due to T. Körner and R. Kaufman of nontrivial compact sets of multiplicity (i.e., not in $U$ ).

Theorem. The o-ideal $U$ is not polar.

Proof. There exists (see Theorem 4.6.3 in [4, p. 117]) a compact set $K \notin U$ on which any measure $\mu$ satisfies that $\lim \sup _{n \rightarrow \infty}|\hat{\mu}(n)|=\|\mu\|$. Then it follows by standard arguments that any measure on $K$ is supported by a countable union of Dirichlet sets (a compact set $D$ is said to be Dirichlet if there exists a sequence of characters converging uniformly on $D$ to the constant function 1). Since all Dirichlet sets are in $U$, any measure in the polar band of $U$ is also null on $K$; hence, $K \in\left(U_{0}\right)^{0} \backslash U$ and $U$ is not polar.

8. As we mentioned in the introduction the $\sigma$-ideal $U$ has no Borel basis. Does $U$ have a Borel $p$-basis? 
9. Let $M(U)$ denote the polar band of $U$. It is clear that $M(U)$ is $\Pi_{2}^{1}$, that is, a CPCA set. What is the exact complexity of $M(U)$ ? If $M(U)$ is Borel, then Theorem 6.2 would give a positive answer to Question 8. If $M(U)$ is not Borel (which is more plausible), then this would give a strengthening for a recent result of $\mathbf{R}$. Kaufman [5] proving that the Rajchman measures form a proper subset of $M(U)$.

10. Find an explicit sequence of continuous functions $f_{n}: \mathbf{T} \rightarrow[0,1]$ and a countable ordinal $\xi$ which would give the representation of the standard band $M=M_{0}(\mathbf{T})$ and its orthogonal band, as in the conclusion of Theorem 9.2. Notice that since $U_{0}$ is not $\mathbf{G}_{\delta}$, by Theorem $7.3 M^{\prime}$ is not $\mathbf{G}_{\delta}$; it follows that one cannot have such a representation with the Fréchet filter and so we have necessarily $\xi>0$.

\section{REFERENCES}

1. G. Debs and J. Saint Raymond, Ensembles d'unicité et d'unicité au sens large, Ann. Inst. Fourier (Grenoble) 37 (1987), 217-239.

2. __ Sélections boréliennes injectives, Amer. J. Math. 111 (1989), 519-534.

3. C. Dellacherie and P. A. Meyer, Probabilités et potentiels, vol. 3, Hermann, Paris, 1984.

4. C. C. Graham and O. C. McGehee, Essays in commutative harmonic analysis, Grundlehren Math. Wiss., vol. 238, Springer-Verlag, New York, 1979.

5. R. Kaufman, M-sets and measures, Ann. of Math. (2) 135 (1992), 125-130.

6. A. S. Kechris, Hereditary properties of the class of closed sets of uniqueness for trigonometric series, preprint.

7. A. S. Kechris and A. Louveau, Descriptive set theory and the structure of sets of uniqueness, Cambridge Univ. Press, Cambridge, 1987.

8. A. S. Kechris, A. Louveau, and W. H. Woodin, The structure of $\sigma$-ideals of compact sets, Trans. Amer. Math. Soc. 301 (1987), 263-288.

9. A. Louveau, Recursivity and capacity theory, Proc. Sympos. Pure Math., vol. 42, Amer. Math. Soc., Providence, R.I., 1985, pp. 285-300.

10. __ Sur la génération des fonctions boréliennes fortement affines sur un convexe compact métrisable, Ann. Inst. Fourier (Grenoble) 36 (1986), 57-68.

11. _ The descriptive theory of Borel sets, Springer-Verlag (to appear).

12. A. Louveau and J. Saint Raymond, Borel classes and closed games: Wadge-type and Hurewicz-type results, Trans. Amer. Math. Soc. 304 (1987).

13. R. Lyons, The size of some classes of thin sets, Studia Math. 86 (1987), 59-78.

14. _ A new type of sets of uniqueness, Duke Math. J. 57 (1988), 431-458.

15. Y. N. Moschovakis, Descriptive set theory, North-Holland, Amsterdam, 1980.

Equipe d'Analyse-Boite 186, Université Paris VI, 4, Place Jussieu, 75252 Paris Cedex 05, FRANCE

E-mail address: gad@ccr.jussieu.fr 